\title{
Indikation und Wirksamkeit der einseitigen Fadenoperation
}

A. Langmann, S. Lindner und M. Koch

Augenklinik Medizinische Universität Graz - Strabologie und Kinderophthalmologie

Einleitung: 1972 beschrieb Cüppers erstmals die Fadenoperation als Operationsmethode durch welche Schielwinkelschwankungen gut ausgeglichen werden können.

Methode: Bei 21 Patienten/innen, bei denen ein monolaterales Innenschielen mit Amblyopie am schielenden Auge vorlag, wurde durch eine einseitige Fadenoperation versucht, den stark schwankenden Schielwinkel $\left(0\right.$ bis $25^{\circ}$ median 0 bis $15^{\circ}$ ) zu beheben. Über Dosierung, Ergebnis und Verlauf über 1 bis 3 Jahre sowie subjektive Zufriedenheit der Patienten/innen wird berichtet. Überprüft wurden die Ergebnisse am See Kid. Diskutiert werden sollten alternative Operationsmethoden.

Zusammenfassung: Die einseitige Fadenoperation bei monolateralem Schielen mit Amblyopie und stark schwankendem Schielwinkel stellt bei Patienten, die jeden Eingriff am gesunden Auge ablehnen, eine Operationsmöglichkeit mit gutem Erfolg dar.

\section{Einfluss drehmomentmindernder Operationen auf den Nahwinkel \\ $S$. Priglinger, R. Hörantner und B. Neudorfer \\ Linz}

Zusammenfassung. Der Einfluss der Hebelarmreduktion der Fadenoperation einerseits und jene der Teilungsoperation auf den Nah- und Fernwinkel andererseits, wird verglichen. 98 Patienten, die wegen schwankendem Schielwinkel an beiden musculi recti mediales teilungsoperiert wurden und auch einen größeren Schielwinkel in der Nähe zeigten, wurden ausgewählt.

Das Drehmomentverhältnis war im Vergleich zu fadenoperierten Patienten für die Nahtentfernung an der Grenze, bzw. außerhalb des Einflusses auf den Nahwinkel. Allerdings war die Differenz der errechneten Länge zwischen teilungsoperierten und fadenoperierten Patienten bei den teilungsoperierten Patienten etwas größer. Diese 98 Patienten wurden nun mit einer randomisierten Gruppe fadenoperierter Patienten verglichen.

Das Ergebnis dieses Vergleiches zeigt, dass die teilungsoperierte Gruppe ein gleich effektives Ergebnis auf den
Nahwinkel aufweist, wie die fadenoperierten Patienten. Bej 5 Patienten der teilungsoperierten Patienten, die nur ein Drehmomentverhältnis 1,2 bis 1,26 aufwiesen, und jene die einen Teilungswinkel zwischen $70^{\circ}$ und $98^{\circ}$ zeigten, hatten ebenfalls einen ausreichenden Effekt auf den Nahwinkel. Diese Studie weist daher nach, dass die Wirksamkeit der Teilungsoperation auf den Nahwinkel gegeben ist. Die Inkongruenz zwischen dem tatsächlichen Operationseffekt auf den Nahwinkel und dem gewählten Drehmoment bei der Teilungsoperation spricht für einen Feed-back-Mechanismus.

Als Reaktion auf die Längendifferenz einerseits, und Spannungsdifferenz andererseits, mögen propriozetive Rückmeldungen, übermittelt durch Palisadenendungen, bzw. durch Muskelspindeln, einen Einfluss auf den postoperativen. Winkel ausüben.

Damit wäre auch der noch Monate später zu beobachtende Trend in die Divergenz zu erklären.

\section{Pulleys, Anatomie und Funktion \\ R. Hörantner, S. Priglinger, M. Buchberger, T. Kaltofen und T. Haslwanter}

Seit etwas mehr als 10 Jahren sind Pulleys als anatomische Strukturen bekannt. Durch die Beschreibung dieser Strukturen wurde das Verständnis der Wirkung der Augenmuskeln wesentlich verändert. Augen- und Orbitamodelle können seit dieser Zeit exakter und besser verwirklicht werden, so auch das Modell SEE++ der UAR Hagenberg.

Einen weiteren Aspekt der Pulleys stellt die mögliche chirurgische Veränderung zur Behebung von Schielsituationen dar. Die Pulleychirurgie wird in letzter Zeit einerseits zunehmend öfter als Therapiemöglichkeit von Schielsituationen beschrieben, andererseits werden bekannte Operationstechniken durch die Berücksichtigung der Pulleys neu bewertet.
In Simulationen mit dem SEE++-System werden verschiedene Pulleyveränderungen an einem geraden Augenmuskel vorgenommen und der Effekt im Hess-Diagramm dargestellt. Weiters wird die durch die Pulleys neu beurteilte Cüppers-Operation auf verschiedene Weise simuliert.

Die Pulleys erweisen sich in der Simulation als sehr stabiles mechanisches System, welches durch Veränderung an nur einem Pulley nur wenig Effekt im Bezug auf die Motilität zeigt. Erst bei einer Veränderung von mehr als einem Pulley wird auch das Motilitätsbild im Hess-Diagramm wesentlich beeinflusst.

Bei der Cüppers-Technik ist rein modellmäßig der Pulleyeffekt eher von geringer Bedeutung. 